\title{
High-resolution Contrast-enhanced CISS for Detailed Anatomical Imaging of the Skull Base
}

Carlos Zamora, MD, PhD, ${ }^{1}$ Edgar Zamora, $\mathrm{MD}^{2}$

${ }^{1}$ University of North Carolina at Chapel Hill, NC, ${ }^{2}$ Montefiore Medical Center, The Bronx, NY

\section{PURPOSE}

- Familiarize the reader with the utility of postcontrast CISS.

- Provide an illustrative review of anatomy and select pathology.

\section{APPROACH/METHODS}

- Retrospective review of cases from our teaching files.

\section{DISCUSSION}

Constructive interference in steady state (CISS)_technical aspects

- Balanced steady state GRE sequence.

- High contrast between CSF \& neurovascular structures.

- Our routine sequence: $0.6 \mathrm{~mm}$ isotropic, can $\sqrt{ }$ to $0.4 \mathrm{~mm}$ or less if needed (e.g. CNIV) but trade-off with acquisition length and SNR.

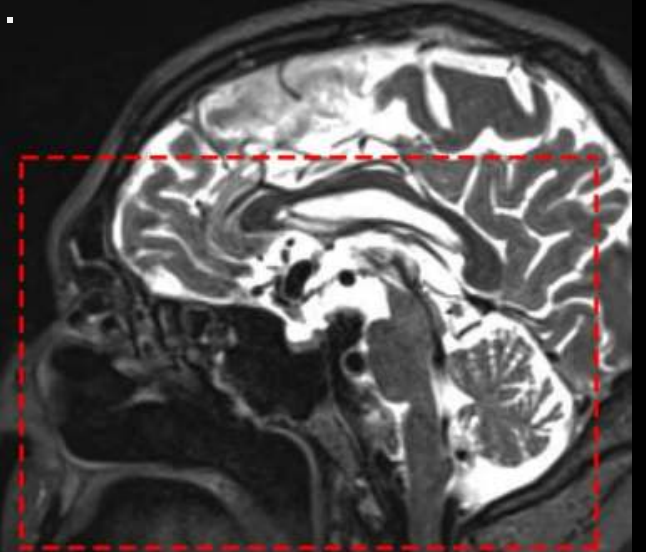

COVERAGE FOR SKULL BASE

- Heavily T2 weighted but also some T1 weighting which provides 2 main advantages after IV contrast administration:

(A) enhancement of venous plexuses, dural/cavernous sinuses.

(B) pathologic enhancement (e.g. neoplasia).

- 3.0T is preferred but most applications can use $1.5 \mathrm{~T}$ if needed.

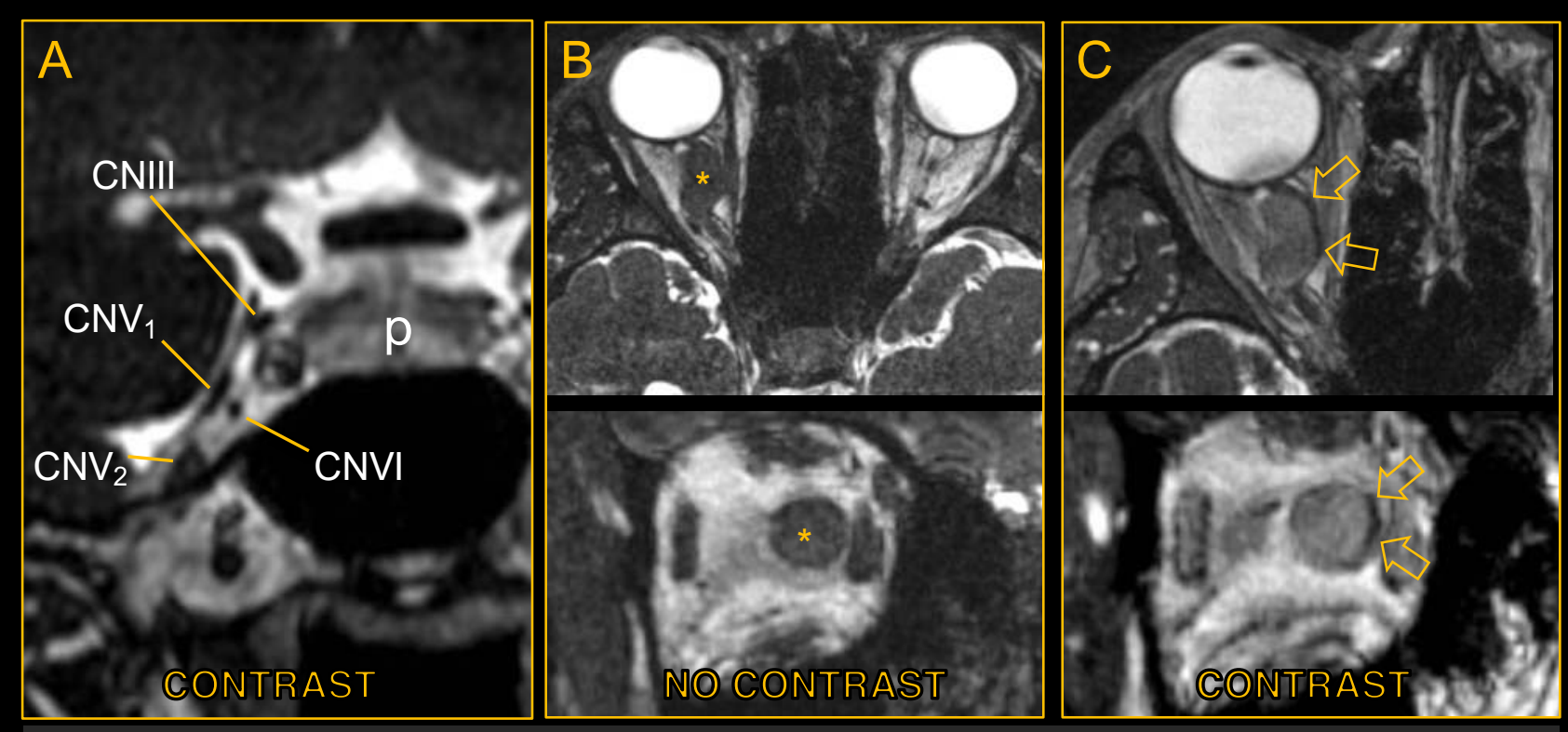

Coronal postcontrast CISS (A) at the level of the pituitary (p) shows dark cranial nerves that are visible against the background of a brightly enhancing cavernous sinus. Noncontrast CISS, different patient (B), shows a right orbital mass (asterisk) initially thought to be a glioma. Postcontrast CISS (C) shows the mass (presumed meningioma) to be extrinsic to the optic nerve. The latter appears dark, flattened \& displaced (arrows).

\section{Central skull base-anatomic considerations}

- Small and complex anatomical region housing several critical neurovascular structures.

- Lesions of the central skull base can affect anterior circulation by encasing ICAs and lead to neuropathies due to cranial nerve (CN) involvement; several potential routes of perineural spread.

- Cavernous sinuses: lined by meningeal (inner) layer of dura, transmit CNIII, CNIV, $\mathrm{CNV}_{1}, \mathrm{CNV}_{2}$ \& CNVI.

- Cavernous/dural sinuses enhance avidly on CISS after contrast $\rightarrow$ allows visualization of CNs beyond cisternal segment.

- CNVI-most medial within the cavernous sinus and most common cause of neuropathy due to central skull base lesion.

- CNIV-thinnest, difficult to image, optimal slice $\leq 0.5 \mathrm{~mm}$ thick.

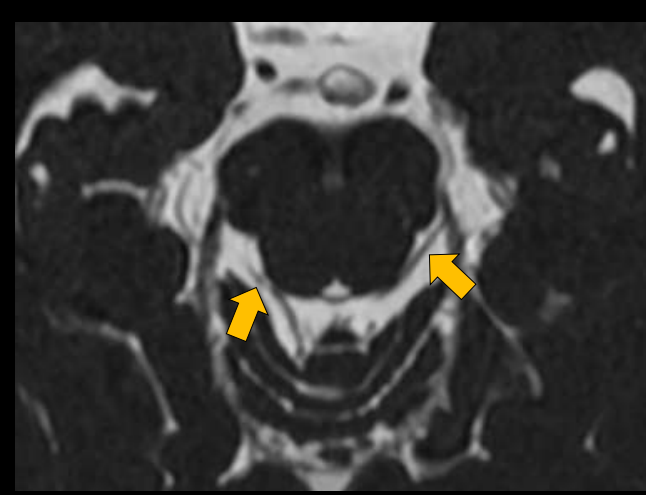

Left: Axial CISS showing cisternal CNIV on the left and partially on the right. Right: Coronal post-

contrast CISS showing enhancing pituitary $(p)$ \& cavernous sinus anatomy.

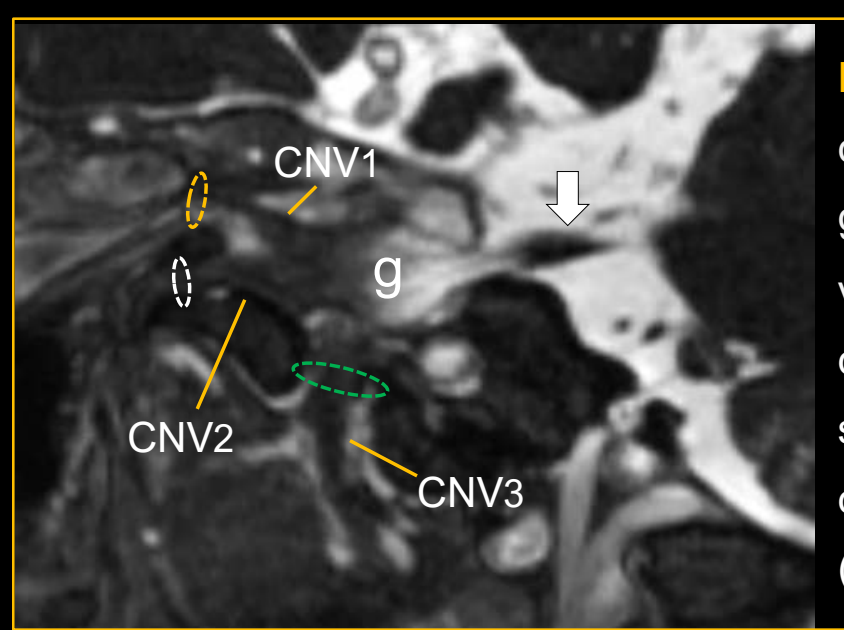

Left: Anatomy of CNV. Sagittal oblique postcontrast CISS at the level of the trigeminal ganglion (g). Cisternal segment is partially visible (arrow). The $3 \mathrm{CNV}$ branches can be delineated due to bright enhancement of the surrounding venous plexuses. Note superior orbital fissure (orange oval), foramen rotundum (white oval), and foramen ovale (green oval).

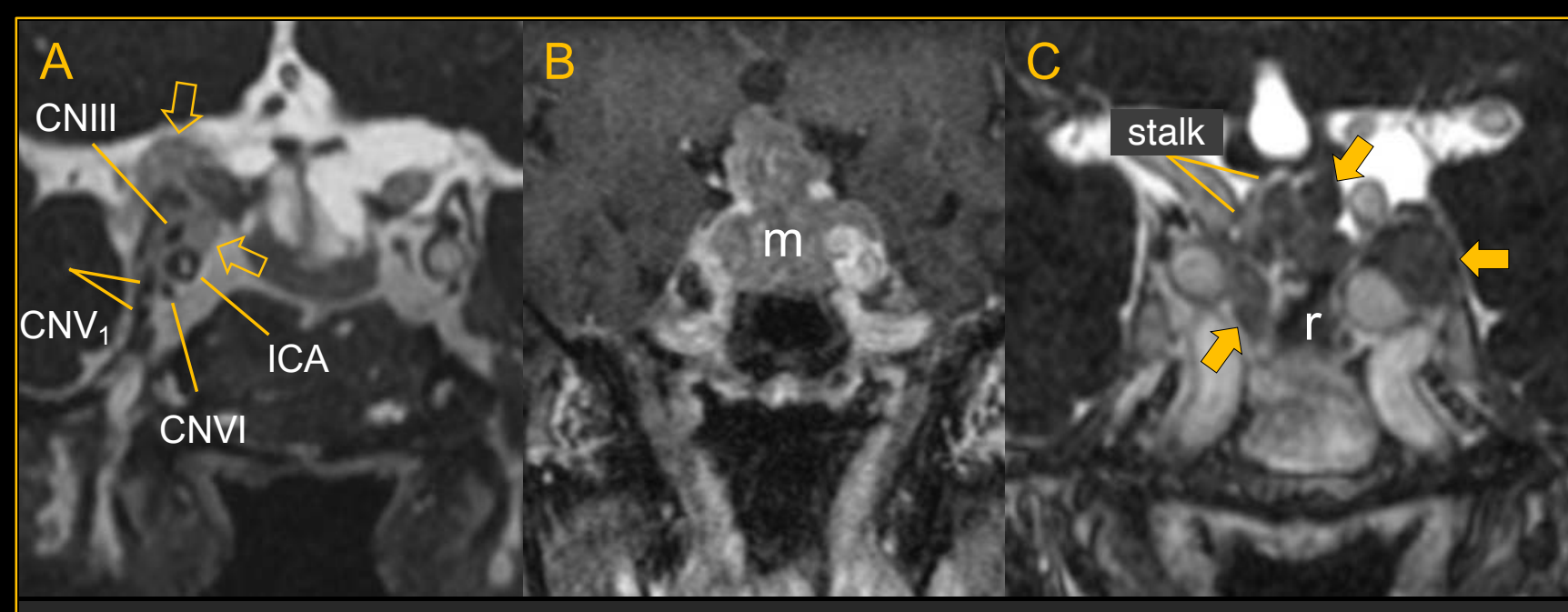

Pituitary adenomas. Coronal postcontrast CISS (A) at the level of the pituitary shows a paraclinoid meningioma (arrows) invading the right cavernous sinus, completely encasing CNIII and partially encasing CNV1, CNVI, and ICA which is narrowed. Coronal postcontrast T1 VIBE in a different patient $(B)$ shows a macroadenoma $(\mathrm{m})$ with suprasellar extension and left cavernous sinus invasion with carotid encasement. Postcontrast CISS (C) following partial resection shows the resection cavity $(r)$ and clearly delineates residual tumor which appears dark (arrows). Note bright pituitary stalk displaced to the right.

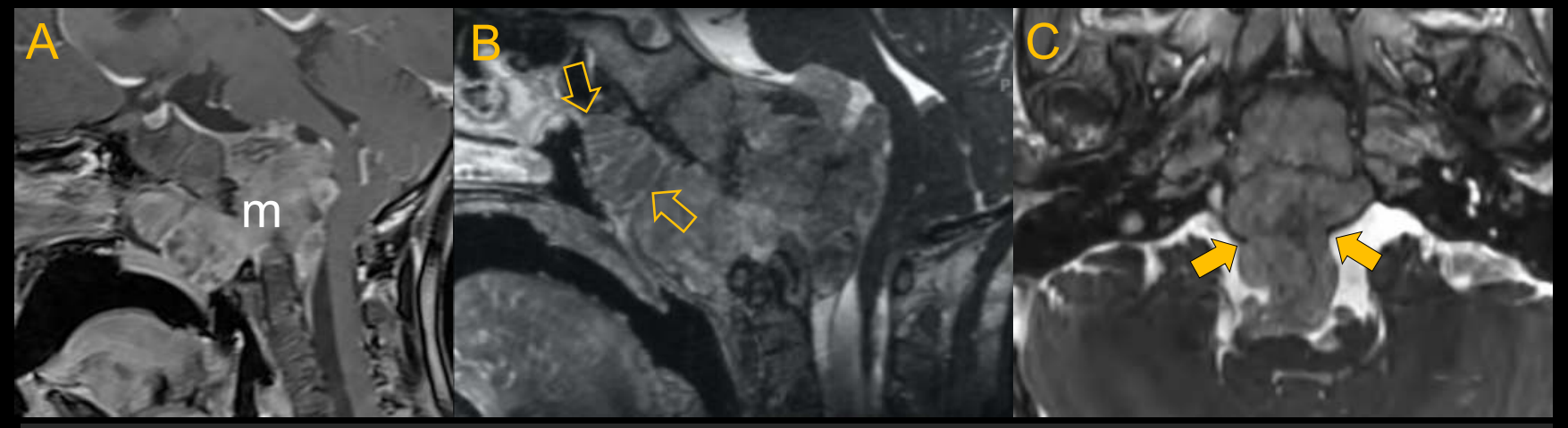

Chordoma. Sagittal postcontrast T1 (A) shows a large enhancing mass $(\mathrm{m})$ centered at the inferior clivus with extension into the posterior fossa and mass effect on the brainstem. Sagittal postcontrast CISS (B) is able to separate the mass from normal adenoid tissue which demonstrates a classic "tigroid" appearance (arrows). Axial post-contrast CISS (C) shows dural rupture (arrows) and extrusion of tumor into the subarachnoid space.

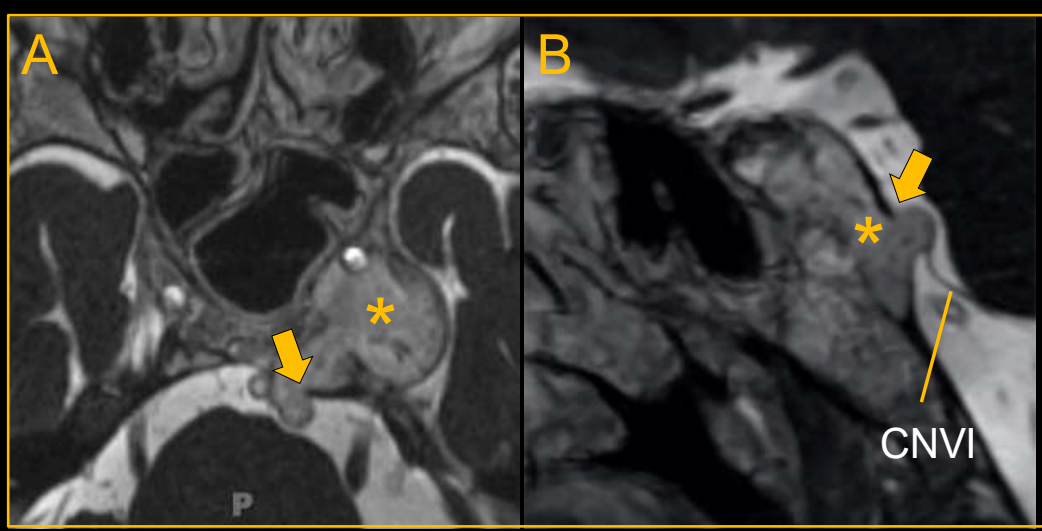

Chondrosarcoma. Axial (A) \& sagittal (B) post-contrast CISS show a mass centered in the left cavernous sinus (asterisk) with dural rupture (arrows), extrusion into the subarachnoid space, and displacement of $\mathrm{CNVI}$.

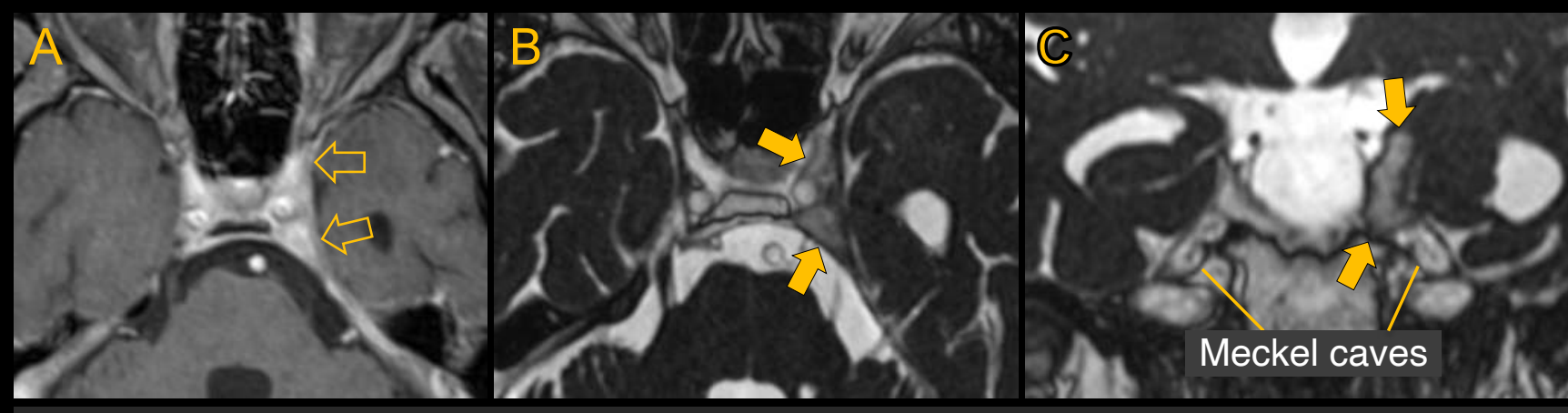

Trigeminal schwannoma. Axial postcontrast T1 VIBE (A) shows abnormal thickening of the left cavernous sinus (arrows). Axial (B) and coronal (C) postcontrast CISS delineate the extent of the tumor which invades the left cavernous sinus and upper aspect of Meckel cave. The tumor can be discriminated against the avidly enhancing cavernous sinus and the CSF-filled Meckel cave.

\section{SUMMARYICONCLUSIONS}

- CISS is a heavily T2-weighted sequence that is also able to show contrast enhancement secondary to some T1 weighting.

- Postcontrast CISS and analogue sequences (e.g. FIESTA-C) can provide exquisite delineation of skull base anatomy \& lesion extent with high spatial and contrast resolution. 